\title{
Application of a new friction theory to ice and snow
}

\author{
LaSSE MaKkONEN \\ Technical Research Centre of Finland, VTT 402150 Espoo, Finland
}

\begin{abstract}
A new theory, in which friction is interpreted as the energy flux required to form surface at contact asperities, is applied to sliding on ice and snow. The results of this theoretical investigation show that in dry friction the relevant contact areas are of almost molecular scale. The properties of the interface layer in ice and snow friction are poorly known, so that the implications of this new theory are somewhat speculative. However, qualitative agreement with experimental data is good, and the theory provides explanations to the success of some empirically developed methods of improving the frictional properties of skis and sledges.
\end{abstract}

\section{INTRODUCTION}

Friction on ice and snow is of fundamental importance in driving safety, icebreaking, design of offshore structures and winter sports, as examples. As reflected by recent extensive reviews on the problem (Forland and Tatinclaux, 1985; Glenne, 1987; Colbeck, 1992), there is still considerable uncertainty about the mechanisms of ice and snow friction.

It is, however, quite generally agreed that the melting of the interface caused by frictional heating is the dominant mechanism. This idea, originally suggested in 1924 by K.B. Veinberg (Deryagin, 1986) and later independently by Bowden and Hughes (1939), has been supported by direct evidence of melt water when objects slide on ice (Tusima and Yosida, 1969). Calculations of the thickness of the melt water film, assuming it behaves as a Newtonian fluid, give rather low values (much smaller than the assumed roughness of ice). This has led various researchers to different conclusions. Some assume that solid to solid contact remains at a considerable portion of the true contact area (e.g. Evans and others, 1976), others that the film behaves as a plastic body with definite slip planes (e.g. Deryagin, 1986) or that the film is only softening but not melting (Akkok and others, 1987).

In any case, it is clear that frictional heating under typical conditions raises the temperature at the points of real contact very close to $0^{\circ} \mathrm{C}$, and that this temperature cannot be exceeded. This allows calculation of the heat flux away from the interface. This heat flux equals the power required to overcome the frictional resistance. Thus when the ice is initially dry and the film temperature is close or at $0^{\circ} \mathrm{C}$, friction is thermally controlled - a fact that makes it possible to calculate the friction coefficient $\mu$ indirectly based on thermal properties of the materials (e.g. Evans and others, 1976; Oksanen and Keinonen, 1982; Akkok and others, 1987; Colbeck, 1988; Lehtovaara, 1989).

Theoretical models of ice and snow friction based on the thermal control mechanism have produced interesting and useful results. However, these theories include many assumptions. Also, questions, such as how the heat is generated in the contact area and what is the mechanism that originally produces frictional resistance are not answered by these theories. If a significant solid to solid contacts remains, additional assumptions on the morphology of the interface are required. Assumptions of this kind are largely unfounded as long as the theory cannot specify the scale at which the frictional mechanisms operate.

In this paper a new concept of the mechanism that generates solid to solid friction is applied to ice and snow. By this, an attempt is made to give a deeper insight into the frictional mechanism on ice, and to estimate the relevant scale of the frictional process.

\section{THEORY}

Consider a situation in Figure 1, where an asperity of a slider of material 1 moves at a velocity $V$ on a much larger asperity of material 2. When 1 moves to the right in Figure 1, at A surface 1, 2 is replaced by 2 and at B surface 2 is replaced by 1,2 . Forming a surface requires a

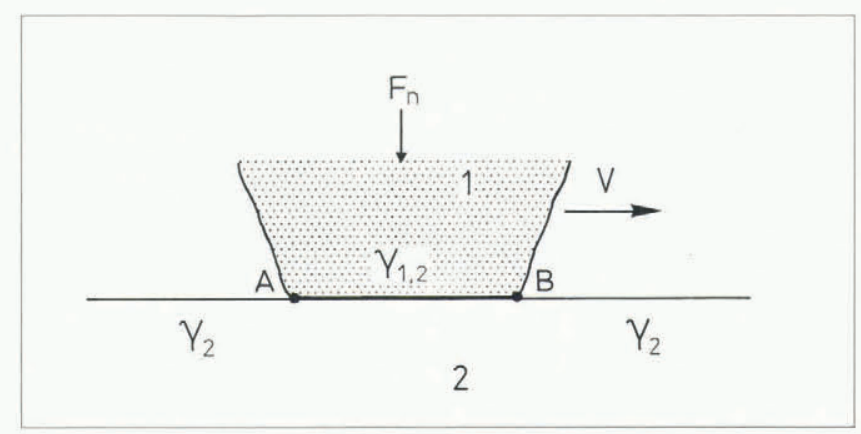

Fig. 1. Schematic figure of a sliding conlact. 
certain amount of energy per unit area. This is the surface energy $\gamma$ resulting from molecular attraction forces. When $\gamma_{2}>\gamma_{1,2}$ in Figure 1 energy is required at $\mathrm{A}$ and energy is released at $\mathrm{B}$. (When $\gamma_{2}<\gamma_{1,2}$ then the reverse is the case.) These energy fluxes are of the opposite sign but of the same magnitude. The energy forced by the motion to be released at B can only be transformed into heat and does not affect the mechanics of the system. Consequently, the free energy required at $\mathrm{A}$ to convert surface 1,2 into surface 2 must be taken from the kinetic energy of the motion. This causes a resistance to motion, i.e. frictional force.

The energy flux required at each contact asperity to convert surface 1,2 into 2 is the surface production rate times the surface energy difference, i.e. $l V\left|\gamma_{2}-\gamma_{1,2}\right|$, where $l$ is the width of the contact. If a slider of the material 1 has $N$ asperities the total energy flux required by the surface generation process is $N l V\left|\gamma_{2}-\gamma_{1,2}\right|$. In pure kinetic friction this energy flux is the only resistance to the motion and, therefore, equals the power $F V=\mu F_{\mathrm{n}} V$ that is supporting the motion. Thus:

$$
\mu=\frac{N l\left|\gamma_{2}-\gamma_{1,2}\right|}{F_{\mathrm{n}}}
$$

The normal force is supported by the true contact area $N l d$ (i.e. by $N$ asperities with the width $l$ and length $d$, when the asperities are assumed to be rectangular), so that the contact pressure is $P_{\mathrm{n}}=F_{\mathrm{n}} / N l d$. As customary (Bowden and Tabor, 1950), one may assume that the materials yield so that $P_{\mathrm{n}}$ is equal to the indentation hardness $H$ of the softer material. This results in $F_{\mathrm{n}}=H N l d$. When this is inserted into Equation (1) both the normal force $F_{\mathrm{n}}$ and the asperity width $l$ disappear giving:

$$
\mu=\frac{\left|\gamma_{2}-\gamma_{1,2}\right|}{H d}
$$

The configuration in Figure 1 is in order to demonstrate the physics of the genesis of friction in a simple way. In reality, the asperities are not rectangular and uniform. This, however, only brings an additional numerical factor into Equation (2). In more general terms $d$ then represents the mean length of the contacts. More importantly, the asperities of material 2 may approach the scale of those of material 1 . In such a configuration there are three types of contacts, those such as in Figure 1, those that require energy both at the front and at the back, and those that release energy both at the front and at the back. For such a geometry the friction coefficient can be shown to be

$$
\mu=\frac{1}{H}\left(\frac{1}{d_{1}}+\frac{1}{d_{2}}\right)\left(\frac{d_{1}}{d_{2}} \gamma_{1}+\left|\gamma_{2}-\gamma_{1,2}\right|\right),
$$

where $\gamma_{1}$ is the surface energy of material 1 and $d_{1}$ and $d_{2}$ are the asperity lengths of the materials 1 and 2 defined in such a way that $d_{1} \leq d_{2}$.

Suppose that material 1 is ice and material 2 is harder than ice. Further suppose (as generally done in physical chemistry) that the surface energies of the solid and the melt are approximately the same (i.e., $\mathrm{l}=\mathrm{i}=\mathrm{w}$, where $\mathrm{i}$ refers to ice and $w$ refers to water). The Young-Dupré equation gives $\gamma_{1,2}+\gamma_{1} \cos \Theta=\gamma_{2}$, where $\Theta$ is the static contact angle of a sessile drop of water on material 2 . Using this in Equation (3) results in:

$$
\mu=\frac{\gamma_{\mathrm{i}}}{H_{\mathrm{i}}}\left(\frac{1}{d_{\mathrm{i}}}+\frac{1}{d_{2}}\right)\left(\frac{d_{\mathrm{i}}}{d_{2}}+|\cos \Theta|\right) .
$$

Equation (4) is to demonstrate that $\mu$ can be estimated using a measurable material property $\Theta$ instead of the surface energies $\gamma_{2}$ and $\gamma_{1,2}$.

When ice slides on ice Equations (3) and (4) read

$$
\mu=\frac{4 \gamma_{\mathrm{i}}}{H_{\mathrm{i}} d_{\mathrm{i}}} \text {. }
$$

\section{DISCUSSION}

Equations (4) and (5) indicate that dry friction increases with increasing surface energy of the materials and decreases with increasing hardness of the softer material. A static contact angle of $90^{\circ}$ of water on a material on which ice is sliding results in minimum friction, according to Equation (4). The theory further shows that friction increases with decreasing length of contact asperities. However, decreasing the ratio $d_{1} / d_{2}$ may increase or decrease $\mu$ depending on the contact angle. It is noteworthy that the true area of contact is not required by this theory, and that when $d$ is a constant the theory obeys Amonton's law ( $\mu$ is independent of $F_{\mathrm{n}}$ ) and Coulomb's law ( $\mu$ is independent of $V$ ).

It is clear that in order to apply this theory to ice we need to know more about the contact length $d$ in Equations (2)-(5). For solving $d$ from Equation (5) the following rough estimate may be used at about $-60^{\circ} \mathrm{C}$ (where ice on ice friction is non-lubricated); $\gamma_{\mathrm{i}} \sim 10^{-1}$ $\mathrm{Jm}^{-2}, \quad H_{\mathrm{i}} \sim 3 \cdot 10^{8} \mathrm{~Pa}$ and $\mu_{\mathrm{i}} \sim 10^{-1}$. This gives $d_{1} \sim 1.3 \cdot 10^{-8} \mathrm{~m}$, a value which corresponds to $\sim 50$ ice molecules.

It thus appears that in ice/ice contact and in the absence of significant ploughing the relevant scale of frictional process is almost molecular, and possibly related to dislocations in the ice crystal lattice. Accordingly, the theory suggests that larger scale structures, such as roughness and grain size, as well as the true contact area are rather unimportant in friction of ice and snow, at least under the conditions where ice/ice contacts prevail. This prediction of the theory is in agreement with experimental data (e.g. Hobbs 1974, p. 416).

It is known from experiments that friction of ice continuously decreases with increasing temperature. The decrease in the surface energy is too small to explain this in terms of Equation (5). Furthermore, the indentation hardness of ice decreases rapidly with increasing temperature, which, as such, should increase friction according to the theory. Therefore, taking that the theory is right also at temperatures closer to $0^{\circ} \mathrm{C}$, the contact asperity length of ice $d_{\mathrm{i}}$ strongly depends on temperature, so that $d_{\mathrm{i}}$ increases with increasing interface temperature. A value of $d_{i}$ at about $-5^{\circ} \mathrm{C}$ may be estimated by Equation (5) taking $\gamma_{\mathrm{i}} \sim 10^{-1} \mathrm{Jm}^{-2}, H_{\mathrm{i}} \sim 5 \cdot 10^{7} \mathrm{~Pa}$ and $\mu \sim 2 \cdot 10^{-2}$. This gives $d_{\mathrm{i}} \sim 0.4 \mu \mathrm{m}-$ thirty times more than at $-60^{\circ} \mathrm{C}$, but still a value much smaller than the typical scale of roughness. 
The theory indicates that $\mu$ does not depend on $V$ in dry friction. Accordingly, the observed velocity dependence of ice and snow friction is related to changes in the extent and thickness of the melt water film. As to the appearance of frictional melting at the interface, the theory suggests no abrupt or significant change in friction, because the surface tensions of ice and water are similar and the contact load is still supported by the ice having the hardness $H_{\mathrm{i}}$.

Application of this theory to practical situations is not straightforward. As discussed in the Introduction, the significance of solid contacts in ice and snow friction is uncertain. Also, the contact asperity length cannot be quantified at present. Some interesting conclusions can be made, however. From the point of view of skiing and skating, it is necessary to have a slider with minimum friction, and Equation (4) indicates how this should be obtained. The slider should be harder than ice so that $H_{\mathrm{i}}$ is the relevant hardness in Equation (4). Further increase in the hardness of the slider has no effect. Second, the contact angle of water on the slider surface should be close to $90^{\circ}$. Furthermore, the asperity length $d$ should have a high value. Comparison of Equations (4) and (5) shows that a good slider on ice may have smaller friction coefficient than ice on ice. These requirements assessed by the theory are in agreement with experience from ski waxes and other sliding surfaces (e.g. Bowden and Tabor, 1950). It is of particular interest here that directional hot polishing of waxed skis is a common empirically developed method in ski racing. The success of this method can be readily explained by the present theory, since the long polymer molecules can be oriented in such a way that the molecular scale contact length $d$ increases.

\section{ACKNOWLEDGEMENTS}

This work was financially supported by the Academy of Finland.

\section{REFERENGES}

Akkok, M., C. M. McC. Ettles and S.J. Calabrese. 1987. Parameters affecting the kinetic friction of ice. Trans. ASME, 7. Tribology, $\mathbf{1 0 9}(3)$, 553-561.

Bowden, F. P. and T. P. Hughes. 1939. Mechanism of sliding on ice and snow. Proc. R. Soc. London, Ser. A, 172(949), 280-298.

Bowden, F. P. and D. Tabor. 1950. The friction and lubrication of solids. Oxford, Clarendon Press.

Colbeck, S. C. 1988. The kinetic friction of snow. F. Glaciol., 34(116),7886.

Colbeck, S. C. 1992. A review of the processes that control snow friction. CRREL Monogr. 92-2.

Deryagin, B. V. 1986. Mechanical properties of the lubricant boundary layer. Soviet Journal of Friction and Wear, 7(5), 773-779.

Evans, D.C.B., J.F. Nye and K.J. Cheeseman. 1976. The kinetic friction of ice. Proc. R. Soc. London, Ser. A, 347(1651), 493-512.

Forland, K. A. and J. -C. P. Tatinclaux. 1985. Kinetic friction coefficient of ice. CRREL Rep. 85-6.

Glenne, B. 1987. Sliding friction and boundary lubrication of snow. Trans. ASME, 7. Tribology, $\mathbf{1 0 9}(4), 614617$.

Hobbs, P. V. 1974. Ice physics. Oxford, Clarendon Press.

Lehtovaara, A. 1989. Kinetic friction between ski and snow. Acta Polytech. Scand. Mech. Eng. Ser., 93.

Oksanen, P. and J. Keinonen. 1982. The mechanism of friction of ice. Wear, 78, 315-334.

Tusima, K. and Z. Yosida. 1969. Melting of ice by friction. Low Temp. Sci., Ser. $A$ 27, 17-30. [In Japanese with English summary.]

The accuracy of references in the text and in this list is the responsibility of the author, to whom queries should be addressed. 